Kardiologe $2020 \cdot 14: 72-80$

https://doi.org/10.1007/s12181-020-00383-3

Online publiziert: 13. März 2020

(c) Deutsche Gesellschaft für Kardiologie Herz- und Kreislaufforschung e.V. Published by Springer Medizin Verlag $\mathrm{GmbH}$, ein Teil von Springer Nature - all rights reserved 2020

\author{
Christian Frerker ${ }^{1} \cdot$ Helge Möllmann ${ }^{2}$ \\ ${ }^{1}$ Klinik für Kardiologie, Medizinische Fakultät und Uniklinik Köln, Universität zu Köln, Köln, Deutschland \\ ${ }^{2}$ Klinik für Innere Medizin I, St.-Johannes-Hospital Dortmund, Dortmund, Deutschland
}

\title{
Herzklappenerkrankungen 2019
}

sichtlich Sterblichkeit und Schlaganfall mindestens gleichwertig, teilweise sogar überlegen ist, wenn sie über einen transfemoralen Zugang durchgeführt wird. Aufgrund dieser guten Ergebnisse erfolgte in der aktuellen Leitlinie aus dem Jahr 2017 und dem deutschen Kommentar der Leitlinie eine Indikationsausweitung der TAVI auf sämtliche Patienten mit hochgradiger AS mit Ausnahme des Niedrigrisikokollektives $[2,3]$. Für diese Patientengruppe brachte nun das letzte Jahr 2019 die noch offenen Antworten.

Die Evolut Low Risk-Studie randomisierte 1468 Patienten mit einem niedrigen operativen Risiko (STS [Society of Thoracic Surgeons] Score $<3 \%$ Sterblichkeit) zur transfemoralen TAVI oder einem konventionellen AKE [13]. Das mittlere Patientenalter betrug 74 Jahre. Es zeigte sich, dass die TAVI dem chirurgischen AKE hinsichtlich des primären Studienendpunktes (Tod oder schwerer Schlaganfall) nach 2 Jahren nicht unterlegen ist (TAVI vs. AKE: $5,3 \%$ vs. $6,7 \%$ ) [13].

\section{》) Es zeigte sich eine signifikant niedrigere In- Hospital-Sterblichkeit nach TAVI gegenüber dem AKE} für Herzklappenerkrankungen im Jahr 2012 als Therapie für inoperable und Hochrisikopatienten empfohlen wurde. Nachdem die TAVI bei den inoperablen und Hochrisikopatienten gute Ergebnisse gegenüber der konservativen bzw. dem konventionell chirurgischen Aortenklappenersatz (AKE) zeigte, folgten Studien bei Patienten mit intermediärem Risiko. Hierbei zeigte sich, dass die TAVI dem chirurgischen AKE hin-
Die PARTNER 3-Studie zeigte ebenso bei einem Niedrigrisikopatientenkollektiv (STS Score $<4 \%$ ) mit 1000 Patienten und einem mittleren Lebensalter von 73 Jahren, dass die transfemorale TAVI dem AKE nicht unterlegen ist [9]. Hierbei war die TAVI hinsichtlich des primären Studienendpunktes (Tod, Schlaganfall oder Rehospitalisierung) nach 12 Mona- ten sogar überlegen (TAVI vs. AKE: 8,5\% vs. $15,1 \% ; p=0,001$ für Überlegenheit) [9].

Zusätzlich zu den beiden großen randomisierten Studien gibt es eine Arbeit aus dem Deutschen Aortenklappenregister zu diesem Thema. Bekeredjian et al. untersuchten die „In-Hospital“- und 1-Jahres-Ergebnisse bei Patienten mit einem niedrigen operativen Risiko (STS Score $<4 \%$ ) [5]. In diese Analyse wurden insgesamt 20.549 Patienten eingeschlossen. Hiervon erhielten 14.487 Patienten einen AKE und 6062 Patienten eine TAVI. Die überwiegende Anzahl der TAVI-Prozeduren erfolgte über einen transvaskulären Zugang (84\%). Trotz der Definition eines Niedrigrisikokollektives unterschieden sich die Patientencharakteristika zwischen den beiden Gruppen in vielen Parametern deutlich. Die TAVIPatienten waren im Durchschnitt mehr als 10 Jahre älter (TAVI vs. AKE: 79 Jahre vs. 67 Jahre; $p<0,0001)$. Der mittlere STS Score betrug 2,11 $\pm 0,95$ (TAVI vs. AKE: $2,86 \pm 0,72$ vs. $1,79 \pm 0,85 ; p<0,0001)$. Die Implantation eines Herzschrittmachers erfolgte häufiger nach TAVI als nach einem AKE (TAVIvs. AKE: $15,1 \%$ vs. $4,4 \%$; $p<0,0001)$. Aufgrund der signifikanten Unterschiede der Patientencharakteristika erfolgte ein adjustierter Vergleich der Endpunkte. Diese gewichtete Analyse zeigte ein signifikant höheres InHospital Überleben nach TAVI im Vergleich zum AKE (TAVI vs. AKE: $98,5 \%$ vs. $97,3 \% ; p=0,003)$. Dieser Vorteil der TAVI war nach 12 Monaten nicht mehr nachweisbar. Es zeigten sich insgesamt für beide Behandlungsgruppen hohe Überlebensraten (TAVI vs. AKE: 90,0\% vs. $91,2 \% ; p=0,158)[5]$. 
Hier steht eine Anzeige.

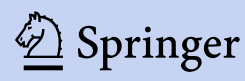



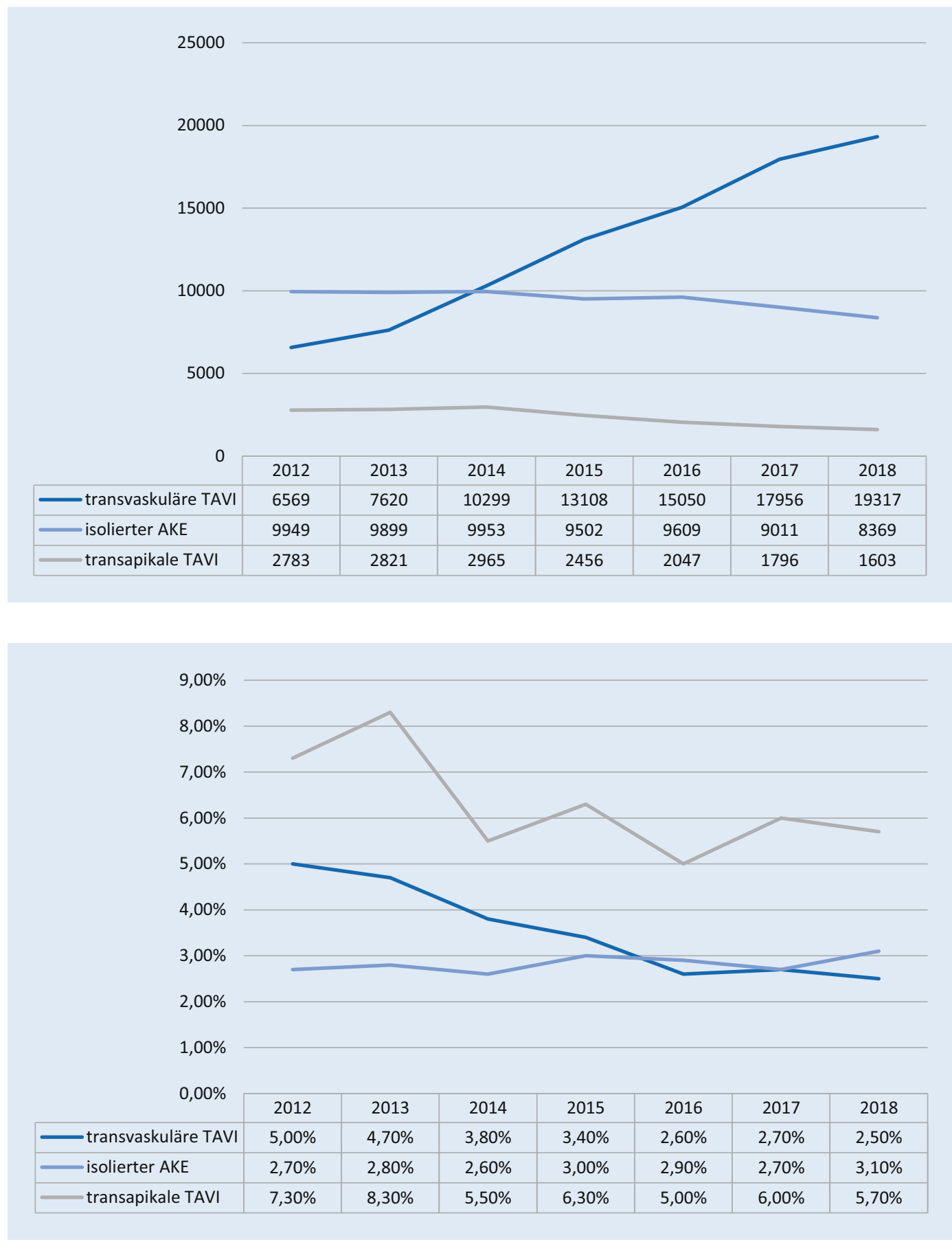

Abb. 1 < Entwicklung der Aortenklappeneingriffe in Deutschland im Zeitraum 2012 bis 2018. TAVI Transkatheteraortenklappenimplantation, AKE konventioneller Aortenklappenersatz
Abb. $2 \triangleleft$ Entwicklung der Krankenhaussterblichkeit in den einzelnen Behandlungsgruppen in Deutschland im Zeitraum 2012 bis 2018. TAVI Transkatheteraortenklappenimplantation, AKE konventioneller Aortenklappenersatz
Nach den einzelnen Studienergebnissen der verschiedenen Risikoklassen wurde im Jahr 2019 eine Metaanalyse mit 8020 Patienten zum Vergleich der TAVI und dem AKE publiziert [14]. Hierbei zeigte sich in einem Nachbeobachtungszeitraum von bis zu 2 Jahren eine signifikant niedrigere Sterblichkeit nach TAVI gegenüber dem AKE (12\%ige relative Risikoreduktion; $p=0,030$ ) [14]. Dieser Überlebensvorteil nach TAVI war konsistent in den 3 verschiedenen Risikogruppen (hoch, intermediär, niedrig). In einer Subgruppenanalyse war v. a. die transfemorale TAVI mit einem Überlebensvorteil (17\%ige relative Risikoreduktion) assoziiert gewesen [14].

Die Ergebnisse der Studien und der Metaanalyse konnten in einer weiteren Real-world-Analyse bestätigt werden. Hierbei wurden alle Patienten, die einen
Aortenklappeneingriff im Jahr 2018 in Deutschland erhalten haben, untersucht [10]. In den letzten 6 Jahren konnte ein deutlicher Zuwachs der transvaskulären TAVI-Prozeduren verzeichnet werden (2012 vs. 2018: 6569 vs. 19.317) [10]. Im gleichen Zeitraum zeigte sich für die transapikale TAVI (2012 vs. 2018: 2783 vs. 1603) und für den konventionellen AKE (2012 vs. 2018: 9949 vs. 8369) ein Abfall der Prozeduren (• Abb. 1). Im 
Jahr 2018 ereignete sich bei 1,9\% der transvaskulären TAVIs eine Gefäßkomplikation. Die Krankenhaussterblichkeit nach transvaskulärer TAVI ging in den letzten Jahren in Deutschland kontinuierlich zurück (2012 vs. 2018: 5,0\% vs. 2,5\%) (• Abb. 2). Im Vergleich zum isolierten $\operatorname{AKE}(3,1 \%)$ und der transapikalen TAVI $(5,7 \%)$ zeigte sich für die transvaskuläre TAVI $(2,5 \%)$ die statistisch signifikant niedrigste Sterblichkeit $(p=0,005)$. Dieser Unterschied war nicht mehr nachweisbar, wenn die Notfalleingriffe (transvaskuläre TAVI vs. AKE: $0,6 \%$ vs. $3,7 \%$ ) in der Analyse rausgenommen werden. In diesem Fall zeigte sich eine Krankenhaussterblichkeit für die transvaskuläre TAVI von $2,5 \%$ gegenüber dem isolierten $\mathrm{AKE}$ von 2,4\% $(p=0,46)$.

Unabhängig von der Risikokalkulation (hohes, intermediäres oder niedriges Risiko) zeigte sich in dieser Arbeit lediglich für die transvaskuläre TAVI über alle Risikogruppen eine niedrigere beobachtete Sterblichkeit gegenüber der erwarteten Sterblichkeit [10].

Zusammenfassend aus allen Studien und Registern zeigt sich heute, dass die TAVI eine etablierte Therapie in der Behandlung der hochgradigen AS unabhängig vom Risiko des Patienten darstellt. Diesbezüglich bedarf es einer erneuten Überarbeitung der Leitlinien hinsichtlich der Indikationsstellung zur TAVI. Die beiden Fachgesellschaften in Deutschland (Deutsche Gesellschaft für Kardiologie und Deutsche Gesellschaft für Thorax-, Herz- und Gefäßchirurgie) erarbeiten derzeit bereits ein gemeinsames Konsensuspapier, das die neuen wissenschaftlichen Aspekte berücksichtigen wird.

\section{Prothesenvergleich}

Bisher existierten 2 randomisierte Studien (CHOICE [Ballon-expandierbare gegenüber selbst-expandierbare Erstgenerations-TAVI-Prothesen] und REPRISE III [mechanisch expandierbare gegenüber selbst-expandierbare Prothese]), die unterschiedliche TAVI-Prothesen miteinander verglichen haben. Im Jahr 2019 wurde nun eine dritte Studie - SCOPE I - zum Vergleich zweier

Kardiologe 2020 · 14:72-80 https://doi.org/10.1007/s12181-020-00383-3

(c) Deutsche Gesellschaft für Kardiologie - Herz- und Kreislaufforschung e.V. Published by Springer Medizin Verlag GmbH, ein Teil von Springer Nature - all rights reserved 2020

\section{Frerker ·H. Möllmann}

\section{Herzklappenerkrankungen 2019}

\section{Zusammenfassung}

Mit zunehmender Alterung der Bevölkerung nimmt die Zahl behandlungsbedürftiger Herzklappenerkrankungen immer weiter zu. In den vergangenen Jahren haben insbesondere die interventionellen Behandlungsoptionen immer weiter an Bedeutung gewonnen. Insbesondere Patienten mit Aortenklappenstenosen können von interventionellen Therapieansätzen profitieren. So ist die Zahl katheterbasierter Eingriffe (Transkatheteraortenklappenimplantation [TAVI]) in Deutschland seit einigen Jahren höher als die klassischer Aortenklappenersatzoperationen. Dies liegt neben dem weniger invasiven Vorgehen v. a. an überzeugenden Studienergebnissen, aber auch an hervorragenden Daten aus der verpflichtenden Qualitätssicherung. Hier konnte gezeigt werden, dass die Mortalität im Krankenhaus bei TAVI-Eingriffen statistisch signifikant niedriger ausfällt. Einen großen Stellenwert hat die interventionelle Therapie auch bei Patienten mit sekundärer Mitralklap- peninsuffizienz. Hier konnte insbesondere in der kürzlich vorgestellten COAPT-Studie ein deutlicher Vorteil der interventionellen Therapie gegenüber der bisherigen alleinigen medikamentösen Therapie nachgewiesen werden. Vergleichsweise neu ist die interventionelle Behandlungsmöglichkeit der Trikuspidalklappeninsuffizienz. Damit ergeben sich wichtige Optionen für viele Patienten, die bislang keiner sinnvollen Therapie zugänglich waren, da isolierte Trikuspidalklappenoperationen bei der regelhaft vorhandenen Komorbidität mit sehr hoher Mortalität verbunden sind. Der vorliegende Beitrag zeigt die wichtigsten Neuigkeiten im Bereich struktureller Herzerkrankungen auf.

\section{Schlüsselwörter}

Aortenklappenstenose $\cdot$ Katheterbasierte Eingriffe - Mitralklappeninsuffizienz . Interventionelle Therapie · Trikuspidalklappeninsuffizienz

\section{Valvular heart disease 2019}

\section{Abstract}

The number of patients suffering from valvular heart diseases is steadily increasing due to the demographic changes and aging of the population. Interventional treatment modalities in particular have gained in importance in recent years. This is especially true for patients with aortic valve stenosis who can benefit from interventional treatment approaches. The number of catheterbased interventions (TAVI) performed in Germany in recent years is higher than the classical surgical aortic valve replacement (SAVR). This is driven not only by convincing results from randomized clinical trials and less invasive approaches but also due to excellent data derived from the mandatory quality assurance. These data could show that the in-hospital mortality was statistically significantly lower for TAVI in comparison to SAVR. The interventional approach has also gained in importance for patients suffering from mitral valve regurgitation secondary to heart failure. The recently presented results of the COAPT trial demonstrated clear superiority of interventional treatment when compared to optimal pharmacotherapy alone. Interventional treatment options for tricuspid valve regurgitation are relatively new. These offer valuable options for many patients for whom a meaningful treatment was so far unavailable, as isolated tricuspid valve surgery is associated with high mortality in this patient group where a high comorbidity is regularly present. This article summarizes the most important new aspects in the field of structural heart diseases.

\section{Keywords}

Aortic valve stenosis - Catheter-based interventions - Mitral valve regurgitation. Interventional treatment - Tricuspid valve regurgitation 
unterschiedlicher TAVI-Prothesentypen vorgestellt [8]. Hierbei wurde die selbstexpandierbare ACURATE neo (Boston Scientific, Marlborough, MA, USA) mit der Ballon-expandierbaren SAPIEN 3 (Edwards Lifesciences, Irvine, CA, USA) verglichen [8]. Es wurden von 5132 gescreenten Patienten im Zeitraum von 2017 bis 2019 insgesamt 739 Patienten an 20 europäischen Zentren eingeschlossen. Es handelte sich um ein typisches TAVIPatientenkollektiv mit einem mittleren Patientenalter von 83 Jahren und einem STS Score von 3,5\%. Hinsichtlich des kombinierten primären Endpunktes nach 30 Tagen konnte die Nichtunterlegenheit der ACURATE neo gegenüber der SAPIEN 3-Prothese nicht erreicht werden (ACURATE neo vs. SAPIEN 3: $24 \%$ vs. $16 \% ; p=0,42$ ). In der Einzelanalyse der Endpunktparameter zeigten sich keine Unterschiede hinsichtlich der Mortalität (2\% vs. $1 \%$ ) und des Auftretens eines Schlaganfalles ( $2 \%$ vs. $3 \%$ ). Demgegenüber war die Inzidenz eines akuten Nierenversagens ( $3 \%$ vs. $1 \%$ ) und einer moderaten oder schweren Aortenklappeninsuffizienz ( $9 \%$ vs. $3 \%$ ) nach TAVI häufiger innerhalb der ACURATE neo-Gruppe [8].

\section{Valve-in-Valve}

Das Konzept der TAVI als Valve-in-Valve bei degenerierter Aortenklappenbioprothese ist heutzutage eine präferierte Therapieform. Hierzu gab es im Jahr 2019 eine weitere Publikation mit 3-JahresErgebnissen aus der PARTNER 2-valvein-valve-Studie und dem folgenden Register [18]. In dieser Analyse handelte es sich um Hochrisikopatienten mit einem mittleren STS Score von 9,1\%. Die 3-Jahres-Sterblichkeit betrug 32,7\%. Es zeigte sich nach 3 Jahren ein mittlerer Gradient über der Aortenklappe von $16,6 \pm 9,0 \mathrm{~mm} \mathrm{Hg}$ und eine Klappenöffnungsfläche von $1,15 \pm 0,42 \mathrm{~cm}^{2}$. Diese Werte waren stabil im Vergleich zur Untersuchung nach 30 Tagen [18]. Diese Ergebnisse mit stabilen Befunden nach 3 Jahren unterstützen somit das Konzept der Valve-in-valve-Therapie bei degenerierten Aortenklappenbioprothesen.
Asymptomatische Aortenklappenstenose

Die bisherige Datenlage zur Therapie einer asymptomatischen AS war bisher kontrovers und dünn. In der Multicenterstudie RECOVERY wurden nun $145 \mathrm{~Pa}$ tienten mit einer asymptomatischen AS zum frühen konventionellen AKE oder $\mathrm{zu}$ einer konservativ beobachtenden Gruppe randomisiert [7]. Das mittlere Patientenalter betrug $64 \pm 9$ Jahre. Eshandelte sich um ein Niedrigrisikopatientenkollektiv mit einem EuroSCORE II von 0,9\%. Die Mehrzahl der Patienten hatte eine bikuspide Aortenklappe (61\%). Echokardiographisch zeigten sich eine mittlere maximale Geschwindigkeit von $5,1 \mathrm{~m} / \mathrm{s}$ über der Aortenklappe und eine Klappenöffnungsfläche von $0,63 \mathrm{~cm}^{2}$. Innerhalb der AKE-Gruppe erhielten jeweils $50 \%$ der Patienten eine mechanische und eine biologische Prothese. Der primäre Endpunkt, definiert als operative Sterblichkeit oder kardiovaskulärer Tod während der Nachbeobachtungszeit, war signifikant geringer für die frühe AKEGruppe (frühe AKE vs. konservativ: 1\% vs. $15 \%$ ). Der mediane Nachbeobachtungszeitraum betrug 6,2 Jahre; $74 \%$ der Patienten innerhalb der konservativen Gruppe erhielten während der Nachbeobachtungszeit im Median von 700 Tagen einen AKE [7].

Diese Daten zeigen, dass der frühe AKE auch bei Patienten mit asymptomatischer AS erwogen werden sollte. Daten zur TAVI-Therapie in diesem Patientenkollektiv sind derzeit noch ausstehend. Hierzu rekrutiert aktuell die EARLY TAVR-Studie in den USA Patienten, und in Deutschland wird in diesem Kontext die EVE TAVI-Studie vorbereitet.

\section{Mitralklappeninsuffizienz}

Im Bereich der AV(atrioventrikular)Klappenerkrankungen hat die DGK (Deutsche Gesellschaft für Kardiologie - Herz- und Kreislaufforschung e. V.) im Jahr 2018 ein Positionspapier publiziert [1]. Hierbei wurden erstmals Qualitätskriterien zur Durchführung von interventionellen Mitralklappeneingriffen definiert [1]. Aufgrund der aktuellen Studienergebnisse ist bereits eine zeitnahe Überarbeitung dieses Positionspapieres geplant.

\section{MITRA-FR und COAPT}

Im Bereich der Mitralklappe sorgte das Jahr 2018 mit den beiden randomisierten Studien MITRA-FR und COAPT für neue Erkenntnisse [12, 16]. Gegenstand beider Studien ist der Nachweis der Sicherheit und Wirksamkeit einer MitraClip(Abbott Structural, Abbott, North Chicago, IL, USA)-Behandlung bei Patienten mit funktioneller Mitralklappeninsuffizienz (MI) gewesen. Die Studienpopulation bestand jeweils aus Patienten mit symptomatischer MI und bereits bestehender optimaler Herzinsuffizienztherapie. Beide Studien hatten einen sehr langsamen Patienteneinschluss. So wurden in MITRA-FR über einen Zeitraum von 3,2 Jahren 2,6 Patienten pro Zentrum und Jahr eingeschlossen [12]. In COAPT betrug die Einschlusszeit sogar 4,8 Jahre mit 1,6 Patienten pro Zentrum und Jahr [16].

Die MITRA-FR-Studie zeigte für den primären kombinierten Endpunkt (Tod oder Rehospitalisierung wegen Herzinsuffizienz) nach $1 \mathrm{Jahr}$ keinen Unterschied zwischen der Interventions- und der Kontrollgruppe (54,6\% vs. $51,3 \% ; p=0,53)$ [12]. Demgegenüber zeigte die COAPT-Studie nach 2 Jahren einen signifikanten Vorteil der MitraClip-Therapie gegenüber einer alleinigen konservativen Herzinsuffizienztherapie hinsichtlich Rehospitalisierung (MitraClip vs. konservativ: $35,8 \%$ vs. 67,9\%; $p<0,001)$ und Sterblichkeit (MitraClip vs. konservativ: $29,1 \%$ vs. $46,1 \%$; $p<0,001$ ) [16]. Die „number needed to treat" für die MitraClip-Therapie betrug 3,1 um eine Rehospitalisation innerhalb von 2 Jahren zu verhindern.

\section{Transkathetermitralklappenersatz}

Bisher existierten für den katheterbasierten Mitralklappenersatz bei relevanter MI lediglich Fallberichte und kleinere Fallserien. Sorajja et al. berichten nun von den ersten 100 Patienten, die über einen transapikalen Zugang (34 oder 36 French) mit der Tendyne-Prothese (Abbott Structural, Abbott, North Chi- 
Hier steht eine Anzeige.

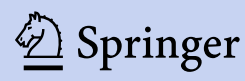




\section{Schwerpunkt}
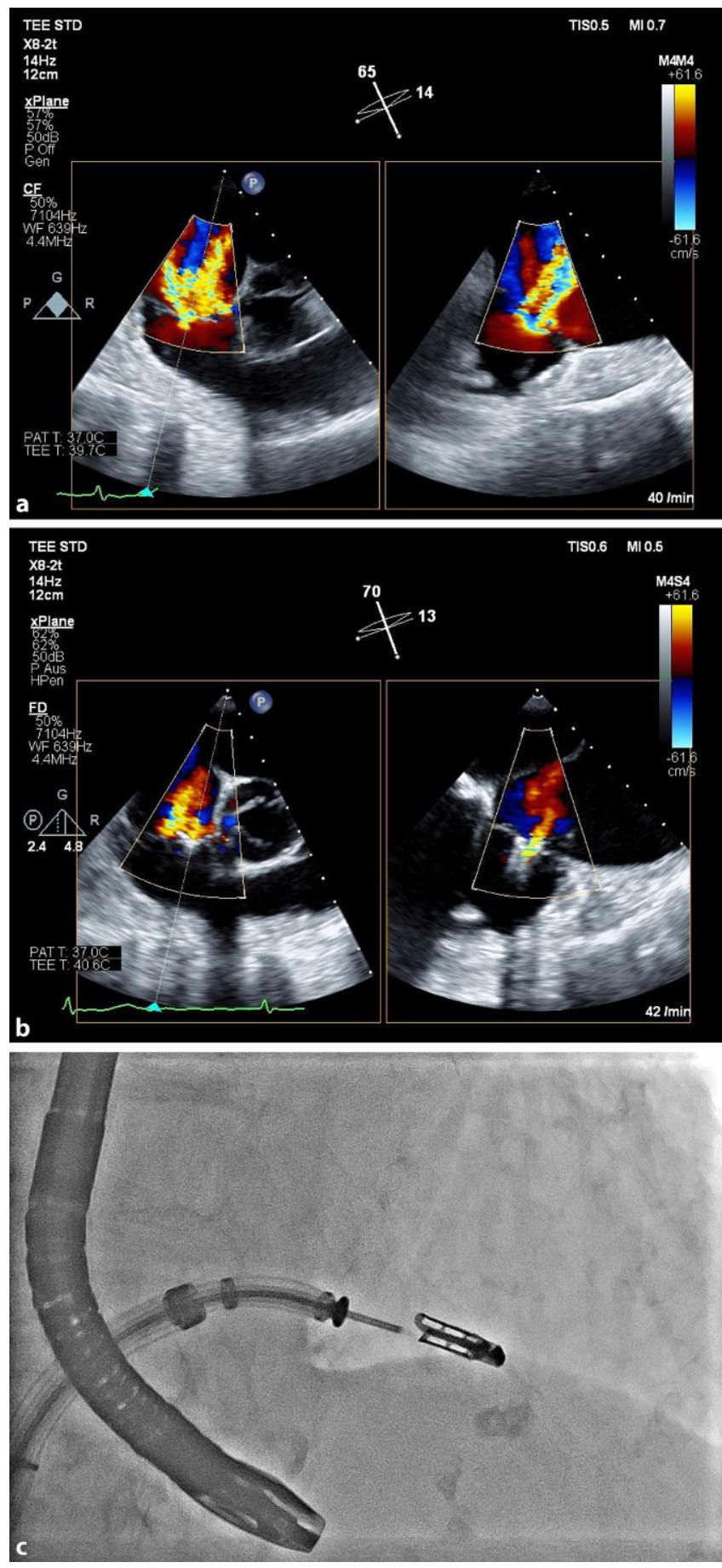

Abb. $3<$ a Schwere Insuffizienz der Trikuspidalklappe bei Anulusdilatation. b Deutliche Reduktion nach Clipimplantation mit typischerweise verbleibender geringer Restinsuffizienz. c Ablösung des Clips in Trikuspidalposition

cago, IL, USA) behandelt worden sind [15]. Das mittlere Patientenalter betrug 75 Jahre mit einem erhöhten operativen Risiko (STS Score 7,8 $\pm 5,7 \%$ ). Die Mehrzahl der Patienten war hochsymptomatisch mit einer NYHA(New York Heart Association)-Klasse III (62\%) oder IV (4\%) und einer reduzierten linksventrikulären Ejektionsfraktion im Mittel von $46 \%$. Die Genese der MI war überwiegend funktionell (89\%). Die technische Erfolgsrate mit erfolgreicher Implantation und Beseitigung der MI betrug $96 \%$ bei einer Prozedurdauer von $136,1 \pm 36,3 \mathrm{~min}$. Die mittlere Nachbeobachtungszeit betrug 13,7 Monate. Das 30-Tages- und 1-Jahres-Überleben betrug 94 und $74 \%$. Echokardiographisch konnte die MI in 98,4\% der Fälle nach 12 Monaten stabil beseitigt wer- den. Klinisch zeigte sich eine deutliche Verbesserung der Belastbarkeit mit einer NYHA-Klasse I (23,3\%) oder II (39,5\%) nach 12 Monaten [15].

\section{Trikuspidalklappeninsuffizienz}

Eine Trikuspidalklappeninsuffizienz (TI) ist eine häufige Erscheinung, insbesondere bei älteren Patienten und bei Vorliegen einer Linksherzklappenerkrankung. Studien haben gezeigt, dass eine moderate oder schwere TI mit einer erhöhten Sterblichkeit verbunden ist. Dennoch wurden Patienten mit einer relevanten TI häufig keiner spezifischen Therapie zugeführt. Dies lässt sich teilweise durch eine hohe Krankenhaussterblichkeit (10,9\%) bei isolierten chirurgischen Eingriffen an der Trikuspidalklappe erklären [4]. Außerdem gelten viele Patienten mit signifikanter TI generell als inoperabel. Erst durch neue interventionelle Verfahren ergaben sich deshalb für dieses Patientengut sinnvolle Therapieansätze.

\section{Interventionelle Therapie im Vergleich zur konservativen Medikation}

Taramasso et al. untersuchten den Einfluss einer katheterbasierten Therapie der TI gegenüber einer konservativ behandelten Vergleichsgruppe [17]. Hierbei dienten die Daten aus dem TriValveRegister als Interventionsgruppe. Das TriValve-Register besteht derzeit aus 472 Patienten aus Europa und Nordamerika, die eine katheterbasierte Therapie der TI erhalten haben. Aufgrund unterschiedlicher Patientencharakteristika und Echokardiographieparameter erfolgte eine Propensity-Match-Analyse mit jeweils 268 Patienten. Eine erfolgreiche Prozedur ( $\mathrm{TI}<\mathrm{Grad} 3$ ) konnte bei $86 \%$ der interventionell behandelten Patienten erreicht werden [17]. Die 6-Monats-Sterblichkeit betrug 13,8 \% für die interventionell behandelte Gruppe gegenüber $26,1 \%$ der konservativ behandelten Gruppe. Dieser Unterschied persistierte auch nach 12 Monaten mit $22,6 \%$ gegenüber $36,2 \%$. Der primäre Endpunkt (Überleben ohne Rehospitalisierung; $p=0,0003$ ) und der sekundäre Endpunkt (absolutes Überleben; 
$p=0,0009)$ wurden signifikant häufiger in der interventionell behandelten Gruppe gegenüber der konservativen Therapie erreicht [17]. Diese Daten zeigen, dass eine interventionelle Therapie bei Patienten mit einer relevanten TI eine gute und sinnvolle Therapieoption darstellt.

\section{Ergebnisse der TRILUMINATE- Studie}

Das Ziel der TRILUMINATE-Studie ist der Nachweis der Sicherheit und der Wirksamkeit eines „edge-to-edge "katheterbasierten Therapieverfahrens in der Behandlung von Hochrisikopatienten mit einer symptomatischen moderaten oder schweren TI gewesen [11]. Hierbei kommt der TriClip (Abbott Structural), der dem MitraClip (Abbott Structural, Abbott, North Chicago, IL, USA) sehr ähnlich ist, zur Anwendung. Die behandelte Patientenkohorte bestand aus 85 Patienten [11]. Das mittlere Patientenalter betrug 78 Jahre mit einem mittleren EuroSCORE II von 8,6\%. Die überwiegende Mehrzahl der Patienten (75\%) war hochsymptomatisch mit einer NYHAKlasse III oder IV. Die Genese der TI war funktionell bei $84 \%$, degenerativ bei $12 \%$ und gemischt bei $4 \%$. Es konnte bei den meisten Patienten eine normale linksventrikuläre Ejektionsfraktion gemessen werden, die im Mittelwert 59\% betrug. Die rechtsventrikuläre Funktion war mit einer TAPSE („tricuspid annular plane systolic excursion") von 14,4 mm reduziert. Es konnte bei $91 \%$ der Prozeduren eine erfolgreiche Implantation mit einer Reduktion der TI um mindestens 1 Schweregrad erreicht werden. Im Durchschnitt wurden 2,2 Clips mit einer durchschnittlichen Prozedurdauer von $153 \mathrm{~min}$ implantiert. In den meisten Fällen erfolgte die Implantation im anterior-septalen Bereich (77\%), gefolgt von posterior-septal (20\%) und anteriorposterior (3\%). Der primäre Sicherheitsendpunkt wurde bei $96 \%$ der Patienten nach 6 Monaten erreicht [11].

Echokardiographisch zeigte sich nach 6 Monaten eine Reduktion der V.-contracta-Weite von $1,73 \mathrm{~cm}$ auf $0,86 \mathrm{~cm}$ $(p<0,0001)$, der effektiven Regurgitationsöffnungsfläche von $0,65 \mathrm{~cm}^{2}$ auf
$0,35 \mathrm{~cm}^{2}(p<0,0001)$ und der Weite des Trikuspidalklappenanulus von $4,33 \mathrm{~cm}$ auf $4,16 \mathrm{~cm}(p=0,0034)$. Klinisch zeigte sich eine Verbesserung mit einem Anstieg der Patienten in einer NYHAKlasse I oder II von $25 \%$ zu Beginn auf $86 \%$ nach 6 Monaten $(p<0,0001)$ [11].

I) Die Therapie mit dem TriClip kann bei Hochrisikopatienten sicher und wirksam durchgeführt werden

Zusammenfassend zeigt sich, dass eine Therapie mit dem TriClip bei Hochrisikopatienten sicher und wirksam durchgeführt werden kann (• Abb. 3).

\section{Fazit für die Praxis}

- Die Behandlung von Herzklappenerkrankungen hat sich durch interventionelle Behandlungsoptionen grundlegend verändert.

- Es werden deutlich mehr Patienten mit Aortenklappenstenosen durch Transkatheteraortenklappenimplantation (TAVI) therapiert als mittels chirurgischen Aortenklappenersatzes (SAVR). Zusammen mit den hervorragenden Ergebnissen sowohl aus randomisierten Studien als auch aus der verpflichtenden Qualitätssicherung wird dadurch ein neuer Goldstandard definiert.

- Auch die Behandlung der Mitralund Trikuspidalklappenerkrankungen erfährt durch katheterbasierte Therapieansätze eine grundlegende Veränderung, von der viele bislang unzureichend behandelte Patienten profitieren können.

\section{Korrespondenzadresse}

Prof. Dr. Helge Möllmann

Klinik für Innere Medizin I, St.-Johannes-

Hospital Dortmund

Johannesstr. 9-13, 44137 Dortmund,

Deutschland

moellmann@me.com
Einhaltung ethischer Richtlinien

Interessenkonflikt. C. Frerker: Vortragshonorare von Abbott, Biotronik, Boston Scientific, Edwards, Medtronic. H. Möllmann: Vortragshonorare/ProctorVergütung von Abbott, Biotronik, Boston Scientific, Edwards Lifesciences

Für diesen Beitrag wurden von den Autoren keine Studien an Menschen oder Tieren durchgeführt. Für die aufgeführten Studien gelten die jeweils dort angegebenen ethischen Richtlinien.

\section{Literatur}

1. Baldus S, Kuck K, Rudolph V et al (2018) Interventionelle Therapie von AV-Klappenerkrankungen - Fokus Mitralklappeninsuffizienz; Positionspapier der Deutschen Gesellschaft für Kardiologie. Kardiologe 12:128-144

2. Baumgartner $\mathrm{H}$, Cremer J, Eggebrecht $\mathrm{H}$ et al (2018) Kommentar zu den Leitlinien (2017) der ESC/EACTS zum Management von Herzklappenerkrankungen. Kardiologe 12:184-193

3. Baumgartner H, FalkV, Bax JJ et al (2017) 2017 ESC/ EACTS guidelines for the management of valvular heart disease. Eur Heart J 38:2739-2791

4. Beckmann A, Funkat AK, Lewandowski J et al (2017) German Heart Surgery Report 2016: the annual updated registry of the German Society for Thoracic and Cardiovascular Surgery. Thorac Cardiovasc Surg 65:505-518

5. Bekeredjian R, Szabo G, Balaban U et al (2019) Patients at low surgical risk as defined by the Society of Thoracic Surgeons Score undergoing isolated interventional or surgical aortic valve implantation: in-hospital data and 1-year results from the German Aortic Valve Registry (GARY). Eur Heart J40:1323-1330

6. Cribier A, Eltchaninoff $H$, Bash A et al (2002) Percutaneous transcatheter implantation of an aortic valve prosthesis for calcific aortic stenosis: first human case description. Circulation 106:3006-3008

7. Kang DH, Park SJ, Lee SA et al (2020) Early surgery or conservative care for asymptomatic aortic stenosis. NEngl J Med 382:111-119

8. Lanz J, Kim WK, Walther T et al (2019) Safety and efficacy of a self-expanding versus a balloonexpandable bioprosthesis for transcatheter aortic valve replacement in patients with symptomatic severe aortic stenosis: a randomised noninferiority trial. Lancet 394:1619-1628

9. Mack MJ, Leon MB, Thourani VH et al (2019) Transcatheter aortic-valve replacement with a balloon-expandable valve in low-risk patients. NEngl J Med 380:1695-1705

10. Mollmann H, Husser O, Blumenstein J et al (2019) Lower mortality in an all-comers aortic stenosis population treated with TAVI in comparison to SAVR. Clin Res Cardiol. https://doi.org/10.1007/ s00392-019-01548-1

11. Nickenig G, Weber M, Lurz $P$ et al (2019) Transcatheter edge-to-edge repair for reduction of tricuspid regurgitation: 6-month outcomes of the TRILUMINATE single-arm study. Lancet 394:2002-2011

12. Obadia JF, Messika-Zeitoun D, Leurent $G$ et al (2018) Percutaneous repair or medical treatment for secondary mitral regurgitation. N Engl J Med 379:2297-2306 
13. Popma JJ, Deeb GM, Yakubov SJ et al (2019) Transcatheter aortic-valve replacement with a selfexpanding valve in low-risk patients. N Engl J Med 380:1706-1715

14. Siontis GCM, Overtchouk P, Cahill TJ et al (2019) Transcatheter aortic valve implantation vs. surgical aortic valve replacement for treatment of symptomatic severe aortic stenosis: an updated meta-analysis. Eur Heart J 40:3143-3153

15. Sorajja P, Moat N, Badhwar V et al (2019) Initial feasibility study of a new transcatheter mitral prosthesis: the first 100 patients. J Am Coll Cardiol 73:1250-1260

16. Stone GW, Lindenfeld J, Abraham WT et al (2018) Transcatheter mitral-valve repair in patients with heart failure. NEngl J Med 379:2307-2318

17. Taramasso M, Benfari G, Van Der Bijl P et al (2019) Transcatheter versus medical treatment of patients with symptomatic severe tricuspid regurgitation. J Am Coll Cardiol 74:2998-3008

18. Webb JG, Murdoch DJ, Alu MC et al (2019) 3-year outcomes after valve-in-valve transcatheter aortic valve replacement for degenerated bioprostheses: the PARTNER 2 registry. J Am Coll Cardiol 73:2647-2655

\section{Stellungnahme der DGK zu COVID-19 und Behandlung mit Hemmstoffen des Renin-Angiotensin-Systems}

Seit einigen Jahren ist bekannt, dass das mit dem Angiotensin-Konversionsenzym (ACE) verwandte ACE2 als Virusrezeptor für den zellulären Eintritt des SARS-CoV aber auch für den neuartigen SARS-CoV2 verantwortlich ist. Für Patienten mit kardiovaskulären Erkrankungen und insbesondere mit Herzinsuffizienz, Diabetes oder der Kombination von beidem besteht wohl eine erhöhte Anfälligkeit für Infektionen und eine erhöhte Sterblichkeit nach Infektion mit SARSCoV2. Experimentelle zellbiologische und tierexperimentelle Daten zeigen, dass die bei diesen Patientengruppen häufig verordneten ACE-Hemmer und Sartane die Aktivität von ACE2 im Herzen erhöhen. Daher kursieren Meldungen, die zu einer Verunsicherung in Bezug auf die Anwendung dieser Medikamente geführt haben.

Alle Spekulationen beruhen auf tierexperimentellen Daten oder Experimenten in Zellmodellen. Es gibt zurzeit keine eindeutigen Hinweise dafür, dass die Gabe von ACE-Hemmstoffen oder Sartanen mit einer erhöhten Sterblichkeit oder Anfälligkeit für Lungenkomplikationen nach SARS-CoV2 assoziiert ist.

Da eine Infektion mit SARS-CoV2 Angiotensin Il erhöht, gibt es auch Spekulationen, dass die Gabe eines Sartans die Anfälligkeit für eine Lungenschädigung senken könnte. Auch dies ist zurzeit spekulativ. Hinweise für eine differenzielle Wirkung unterschiedlicher Hemmstoffe des Renin-Angiotensin-Systems bei Patienten finden sich nicht.

Im Gegensatz dazu zeigen ACE-Hemmstoffe und Sartane eine bewiesene Wirkung bei arterieller Hypertonie und Herzinsuffizienz und sind bei diesen Erkrankungen zur Senkung der Sterblichkeit und der Krankenhausaufnahmewahrscheinlichkeit sowie zum Schutz der Nieren indiziert. Da eine Unterbrechung oder ein Absetzen dieser Therapien zu einer Steigerung der Sterblichkeit und Hospitalisierungsrate führen würde, empfiehlt die DGK, diese Therapien fortzuführen bis weitere $\mathrm{Da}$ ten aus klinischen Studien verfügbar sind. Auch gibt es keine Hinweise dafür, dass eine Umstellung von ACE-Hemmern auf Sartane bei Patienten mit SARS-CoV2-Infektionen günstig ist.

Anmerkungen zur Stellungnahme spiegeln den aktuellen Kenntnisstand wider. Neuere wissenschaftliche Erkenntnisse könnten zu einer Änderung von Empfehlungen führen.

\section{Originalpublikationen:}

1. Ponikowski P et al. ESC Scientific Document Group.2016 ESC Guidelines for the diagnosis and treatment of acute and chronic heart failure: The Task Force for the diagnosis and treatment of acute and chronic heart failure of the European Society of Cardiology (ESC) Developed with the special contribution of the Heart Failure Association (HFA) of the ESC. Eur Heart J 2016;37:2129-2200

2. Williams B et al. 2018 ESC/ESH Guidelines for the management or arterial hypertension: The Task Force for the management of arterial hypertension of the European Society of Cardiology and the European Society of Hypertension: The Task Force for the management of arterial hypertension of the European Society of Cardiology and the European Society of Hypertension. Eur Heart J 2018;39(33):3021-3104.

3. Yang $X$ et al. Clinical course and outcomes of critically ill patients with SARCCoV2 pneumonia in Wuhan, China: a singlecentered, retrospective, observational study. Lancet Respir Med 2020; published online Feb 24. https://doi.org/10.1016/S22132600(20)30079-5.

4. Guan W et al. Clinical caracteristics of coronarvirus disease 2019 in China. N Engl J Med 2020; published online Feb 28. DOI:10.1056/NEJMoa2002032.

5. WWan $Y$ et al. Receptor recognition by novel coronavirus from Wuhan: An analysis based on decade-long structural studies of SARS. J Virology 2020; published online Jan 29. DOI:10.1128/JVI.00127-20.

6. Zheng YY et al. Covid-19 and the cardiovascular system. Nature Reviews 2020; https://doi.org/10.1038/s41459-020-0360-5.

Quelle: Deutsche Gesellschaft für Kardiologie - Herz- und Kreislaufforschung e.V., www.dgk.org 